\title{
Expression level of Pre B cell leukemia homeobox 2 correlates with poor prognosis of gastric adenocarcinoma and esophageal squamous cell carcinoma
}

\author{
YING QIU $^{1,2}$, BOGEN SONG ${ }^{1}$, GUIFEN ZHAO ${ }^{1}$, BIYONG DENG $^{1}$, TOMOKI MAKINO ${ }^{3}$, YASUHIKO TOMITA ${ }^{4}$, \\ JUNCHEN WANG ${ }^{5}$, WENJUAN LUO ${ }^{6}$, YUICHIRO DOKI ${ }^{3}$, KATSUYUKI AOZASA ${ }^{2}$ and EIICHI MORII ${ }^{2}$ \\ ${ }^{1}$ Department of Pathology, Tongji University School of Medicine, Siping Road 1239, Shanghai 200092, P.R. China; \\ Departments of ${ }^{2}$ Pathology and ${ }^{3}$ Gastroenterological Surgery, Osaka University Graduate School of Medicine, \\ 2-2 Yamadaoka, Suita, Osaka 565-0871; ${ }^{4}$ Department of Pathology, Osaka Medical Center for Cancer and \\ Cardiovascular Diseases, 1-3-3 Nakamichi, Higashinari, Osaka 537-8511, Japan; ${ }^{5}$ Department of Pathology, \\ Tongji University Affiliated East Hospital, Jimo road 150, Pudong, Shanghai 200120; ${ }^{6}$ School of Medicine, \\ Xi'an Jiaotong University, No.76 Yanta Weststreet, Xi'an 120, Shanxi 710032, P.R. China
}

Received September 23, 2009; Accepted November 6, 2009

DOI: 10.3892/ijo_00000541

\begin{abstract}
Pre B cell leukemia homeobox 2 (PBX2), a member of PBX family, acts as a co-factor of homeobox proteins to regulate proliferation and differentiation of tumor cells. Our recent study revealed prognostic significance of $\mathrm{PBX} 2$ expression in non-small cell lung carcinoma. The significance of $\mathrm{PBX} 2$ expression was examined in cases with gastric cancer (GC) and esophageal squamous cell carcinoma (ESCC), and the role of PBX2 in tumor behavior was evaluated in GC and ESCC cell lines of knocked-down PBX2 expression. Expression level of PBX2 was immunohistochemically examined in 94 patients of GC and 64 patients of ESCC. Staining intensity for PBX2 was categorized as equal to or stronger (level 1) and weaker (level 2) than that of endothelial cells. Cases with level 1 expression in more than $20 \%$ of tumor were defined as high and others low expression. Patients with low PBX2 expression showed a better prognosis than those with high expression in both GC and ESCC. Multivariate analysis revealed PBX2 expression to be an independent prognosticator for both GC and ESCC. Knocked-down expression of PBX2 in GC and ESCC cell lines resulted in decrease of in vitro colony formation and in vivo tumorigenic activities, but proliferative and invasive activities did not change. Under serum depletion, apoptotic cell proportion was higher in PBX2 knocked-down cells than in control cells. The knock-down of PBX2 reduced Bcl-2 expression. Taken
\end{abstract}

Correspondence to: Dr Eiichi Morii, Department of Pathology, Osaka University Graduate School of Medicine, 2-2 Yamadaoka, Suita, Osaka, 565-0871, Japan

E-mail: morii@patho.med.osaka-u.ac.jp

Key words: Pre B cell leukemia homeobox, gastric adenocarcinoma, esophageal squamous cell carcinoma, prognosis together, the high expression level of PBX2 was an independent negative prognosticator for both GC and ESCC, and $\mathrm{PBX} 2$ might promote tumor growth through suppression of apoptosis.

\section{Introduction}

Pre B cell leukemia homeobox (PBX) family belongs to the superfamily of homeodomain-containing proteins, among which homeobox (HOX) proteins are most intensively investigated. HOX proteins act as transcription factors in cooperation with other regulatory proteins, including PBX2, and are involved in the control of developmental processes and cell differentiation $(1,2)$. Aberrant overexpression of HOX genes has been reported in various kinds of cancers, such as oral squamous cell carcinoma, non-small cell lung carcinoma (NSCLC), ovarian carcinoma, esophageal squamous cell carcinoma (ESCC), gastric carcinoma (GC), hepatocellular carcinoma, prostate cancer, bladder cancer, and cervical cancer, together with acute lymphoblastic leukemia (3-12). Overexpression of HOX proteins is correlated with poor prognosis in some cancers, such as acute myeloid leukemia $(13,14)$. In contrast to other HOX proteins, PBX family proteins have not been studied in detail. The interference of PBX binding to HOX proteins yielded apoptosis and reduced growth activity of tumors in vivo, indicating that the interaction of PBX to HOX appears to be crucial in tumor behavior (15-17).

GC and ESCC are the most common cancers not only in Asia but also in the world. They are highly aggressive carcinomas with poor outcome: annually over 1 million people die of these cancers worldwide (18-20). In Japan and Southern China, 5-year survival rate of GC and ESCC patients is less than $20 \%$, due to the occasional occurrence of systemic metastasis within a few years even after curative resection (21-23). For improvement of prognosis of these patients, it is essential to understand molecular mechanisms underlying 
aggressiveness of GC and ESCC. Our recent study revealed that the NSCLC with high level of PBX2 expression showed a poorer prognosis than that with low expression (24). In the present study, the expression level of PBX2 proteins was immunohistochemically examined in 94 patients with GC and 64 with ESCC, who underwent curative surgery, and its correlation with clinicopathological features and prognosis of patients was analyzed. To evaluate the role of PBX2 in tumor behavior, the effects of knock-down of this gene on colony formation, apoptosis, proliferation and invasive activities in GC and ESCC cell lines were estimated.

\section{Patients and methods}

Patients. Gastric cancer. A total of 94 patients with histologically proven GC were analyzed: they underwent curative resection at the Department of Surgery, East Hospital of Tongji University (Shanghai, China) between 1998 and 2002. Curative resection is defined as the complete resection of gastric lesions, with no remaining tumorous lesions at the postoperative histological examinations. There were 65 males and 29 females, with ages ranging from 32 to 82 (median 64) years. Surgical procedures employed were total gastrectomy in 24 patients, proximal gastrectomy in 6 , distal gastrectomy in 61 , and partial gastrectomy in 3 patients. Size of main tumor at macroscopical examination of the resected stomach ranged from 10 to 200 (mean 28) $\mathrm{mm}$.

Adjuvant chemotherapy was performed in 49 patients (preoperative in one, during surgery in 12, postoperative in 31 , both during surgery and the postoperative period in 5) with a high risk for tumor recurrence, i.e., presence of lymph node metastasis, large tumor size, and tumor invasion to the serosa. Chemotherapeutic protocols were as follows: fluorouracil (5-FU) or its derivative alone in two patients; mitomycin $\mathrm{C}$ (MMC) alone in one; 5-FU and cisplatinum (CDDP) in nine; CDDP and MMC in six; 5-FU and MMC in three; 5-FU, MMC, and CDDP in four; 5-FU, MMC, and doxorubicin (DXR) in three; CDDP, MMC, and etoposide (VP-16) in seven; 5-FU, CDDP, and VP-16 in four; 5-FU, $\mathrm{MMC}$, and epirubicin (EPI) in five; 5-FU, MMC, and cyclophosphamide (CTX) in two; 5-FU, CDDP, and CTX in two; 5-FU, MMC, VP-16, and CTX in one. Follow-up period for survivors ranged from 1 to 81 (median 30.1) months. The study was approved by the institutional review board of East Hospital, Tongji University.

Esopageal cancer. Sixty-four patients underwent surgery for ESCC at the Department of Surgery, Osaka University Hospital during the period from 2000 to 2005. There were 59 males and 5 females with ages ranging from 49-82 (median 65) years. Preoperative endoscopic examination of esophageal lesions was performed, and a histological diagnosis of ESCC was made based on the histological examination of biopsy specimens. Preoperative diagnostic examinations, including esophagography, computed tomography, and ultra-sound were performed for clinical staging of disease. All patients underwent curative surgery for ESCC: the resected esophagus was macroscopically examined to determine the location and size of the tumor. The size of the main tumor ranged from 1 to $390 \mathrm{~mm}$ (median $45 \mathrm{~mm}$ ). None of patients received chemotherapy and radiation therapy before surgical resection.

Follow-up examinations. After surgery, all patients underwent laboratory examinations such as routine peripheral blood cell counts with measurement of the serum squamous cell carcinoma antigen levels in cases of ESCC every 1-6 months, and chest roentgenography, ultrasonography of the liver, computerized tomographic scan of the thorax and abdomen, and endoscopic examination of the remaining esophagus in cases of ESCC at intervals of 6-12 months.

Histological examination. Samples obtained from the gastric or esophageal lesions were fixed in $10 \%$ formalin and routinely processed for paraffin embedding. Histologic sections cut at $4 \mu \mathrm{m}$ were stained with hematoxylin and eosin and immuno-peroxidase procedures. Histologic sections were analyzed by two investigators (Y.Q. and Y.T. for GC, and Y.Q. and E.M. for ESCC) to define the extent and mode of cancer invasion in the stomach or esophagus, lymph node metastasis, and histological subtype. Tumor stage was determined according to the pathologic TNM classification (25).

Immunohistochemistry. Avidin-biotin-peroxidase complex method ( $\mathrm{ABC}$ ) was used for immunostaining. Antigen retrieval was done with heating the sections in $10 \mathrm{mM}$ citrate buffer for 10 min. Rabbit polyclonal anti-human PBX2 antibody (1:200, Santa Cruz, Santa Cruz, CA) was used as primary antibody. After washing with phosphate buffered saline (PBS), the sections were immersed in $2 \%$ hydrogen peroxidase in methanol for 20 min to block endogenous peroxidase activity, incubated with goat non-immune serum for $30 \mathrm{~min}$, then incubated with primary antibody at room temperature for $1 \mathrm{~h}$ and at $4^{\circ} \mathrm{C}$ overnight. Sections were counterstained with hematoxylin. Immunostaining was carried out as described previously (24). Positive staining of endothelial cells in the same section was used as an internal positive control. For a negative control, primary antibody was replaced by nonimmunized rabbit immunoglobulin G serum (Vector Laboratories, Burlingame, CA), giving uniformly negative results.

All immunohistochemically stained sections were examined in a blinded manner without any knowledge of the clinicopathologic parameters and patient outcome. Staining intensity of the tumor cells was compared to that of endothelial cells, and categorized as follows: equal to or stronger (level 1) and weaker (level 2) than that in the endothelial cells. Protein expression level was scored as the percentage of positive cells at 10 high power fields. Cases with level 1 expression in more than $20 \%$ of tumor cells were defined as 'high' and others as 'low' expression. Typical fields for high and low cases of GC and ESCC are shown in Fig. 1A.

Cell culture. Human gastric adenocarcinoma cell line (MNK-45) and human esophageal squamous cell carcinoma cell line (KYSE70) were maintained in Dulbecco's modified Eagle's medium (DMEM, Sigma, St. Louis, MO) supplemented with $10 \%$ fetal bovine serum (FBS; Nippon Bio-supp. Center, Tokyo, Japan; DMEM- $10 \% \mathrm{FBS}$ ) at $37^{\circ} \mathrm{C}$ in a humidified atmosphere containing $5 \% \mathrm{CO}_{2}$. 
Table I. PBX2 expression and clinicopathological factors in 94 patients with GC.

\begin{tabular}{|c|c|c|c|c|c|}
\hline $\begin{array}{l}\text { Clinicopathological } \\
\text { features }\end{array}$ & Category & No. of patients & $\begin{array}{l}\text { PBX2 high } \\
\text { expression } \\
\quad(n=56)\end{array}$ & $\begin{array}{l}\text { PBX2 low } \\
\text { expression } \\
(n=38)\end{array}$ & P-value \\
\hline Age, mean \pm SD & Mean $(64.10 \pm 13.09)$ & & $64.66 \pm 13.35$ & $63.28 \pm 12.67$ & NS \\
\hline \multirow[t]{2}{*}{ Sex } & Male & 65 & 41 & 24 & NS \\
\hline & Female & 29 & 15 & 14 & \\
\hline \multirow[t]{3}{*}{ Tumor location } & Upper third & 23 & 19 & 4 & NS \\
\hline & Middle third & 23 & 10 & 13 & \\
\hline & Lower third & 48 & 27 & 21 & \\
\hline \multirow[t]{2}{*}{ Tumor size, mm } & $<30$ & 68 & 39 & 29 & NS \\
\hline & $\geq 30$ & 26 & 17 & 9 & \\
\hline \multirow{2}{*}{$\begin{array}{l}\text { Histological } \\
\text { differentiation }\end{array}$} & Undifferentiated & 55 & 35 & 20 & NS \\
\hline & Differentiated & 39 & 21 & 18 & \\
\hline \multirow[t]{2}{*}{ Vascular invasion } & Absent & 66 & 40 & 26 & NS \\
\hline & Present & 28 & 16 & 12 & \\
\hline \multirow[t]{2}{*}{ Lymphatic invasion } & Absent & 79 & 45 & 34 & NS \\
\hline & Present & 15 & 11 & 4 & \\
\hline \multirow{4}{*}{$\begin{array}{l}\text { Depth of tumor } \\
\text { invasion }\end{array}$} & pT1 & 15 & 7 & 8 & NS \\
\hline & pT2 & 17 & 8 & 9 & \\
\hline & pT3 & 30 & 17 & 13 & \\
\hline & pT4 & 32 & 24 & 8 & \\
\hline \multirow{4}{*}{$\begin{array}{l}\text { Lymph node } \\
\text { metastasis }\end{array}$} & $\mathrm{pNO}$ & 23 & 13 & 10 & NS \\
\hline & $\mathrm{pN} 1$ & 52 & 31 & 21 & \\
\hline & $\mathrm{pN} 2$ & 15 & 9 & 6 & \\
\hline & $\mathrm{pN} 3$ & 4 & 3 & 1 & \\
\hline \multirow[t]{2}{*}{ Chemotherapy } & Not performed & 45 & 25 & 20 & NS \\
\hline & Performed & 49 & 31 & 18 & \\
\hline
\end{tabular}

GC, gastric cancer.

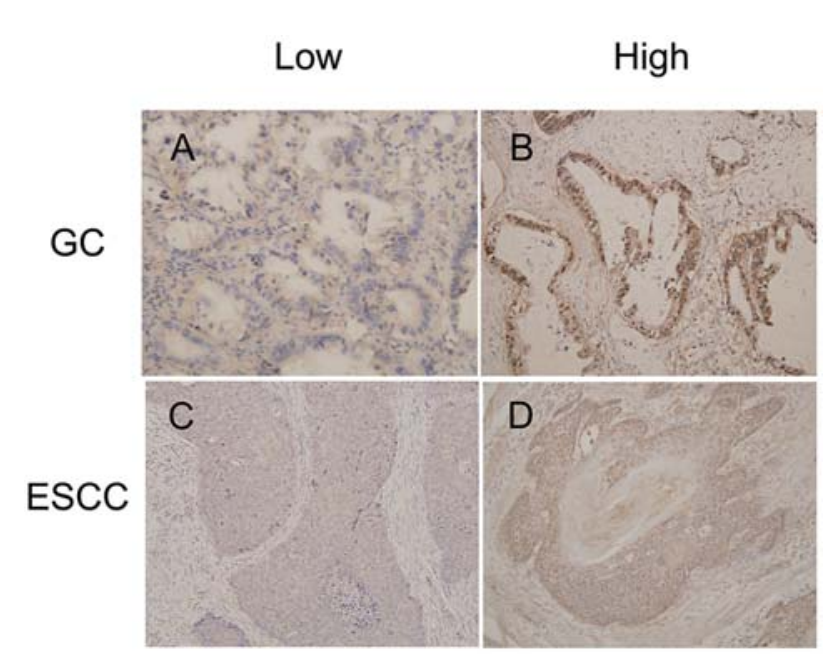

Figure 1. PBX2 expression in GC (A and B) and ESCC (C and D). Low expression in $\mathrm{A}$ and $\mathrm{C}$, and high expression in $\mathrm{B}$ and $\mathrm{D}$. ABC method, $\mathrm{x} 200$.
Construction of PBX2 siRNA plasmids and transfection. To generate a stably expressing si-RNA system, the BLOCK-iT Pol II miR RNAi expression vector kit (Invitrogen, Carlsbad, CA) was used. The plasmids producing siRNA were transfected into the MNK-45 and KYSE70 cells, respectively, with Lipofectamine 2000 reagent (Invitrogen) according to the manufacturer's protocol. Cells stably expressing the PBX2 si-RNA and control cells stably expressing vector alone were established in DMEM-10\% FBS containing blasticidin (Invitrogen) at a concentration of $24 \mu \mathrm{g} / \mathrm{ml}$ in KYSE70 and MNK-45 cells. The knocked-down of PBX2 expression in the stable clones was determined by Western blot analysis.

Western blot analysis. Cells were washed with ice-cold PBS and lysed. Protein concentration was determined using a protein assay reagent (Bio-Rad, Hercules, CA). Equal amounts of proteins were separated on $10 \%$ sodium dodecyl sulfatepolyacrylamide gel electrophoresis (SDS-PAGE) gels and transferred to polyvinylidene fluoride membranes (Millipore, 
Table II. PBX2 expression and clinicopathological factors in 64 patients with ESCC.

\begin{tabular}{|c|c|c|c|c|c|}
\hline $\begin{array}{l}\text { Clinicopathological } \\
\text { features }\end{array}$ & Category & No. of patient & $\begin{array}{l}\text { PBX2 high } \\
\text { expression } \\
\quad(n=21)\end{array}$ & $\begin{array}{l}\text { pPBX2 low } \\
\text { expression } \\
(n=43)\end{array}$ & P-value \\
\hline Age, mean \pm SD & Mean $(66.08 \pm 7.02)$ & & $66.62 \pm 6.09$ & $65.95 \pm 7.21$ & NS \\
\hline \multirow[t]{2}{*}{ Gender } & Male & 59 & 19 & 40 & NS \\
\hline & Female & 5 & 2 & 3 & \\
\hline \multirow[t]{5}{*}{ Tumor location } & Cervix & 2 & 2 & 0 & NS \\
\hline & Upper third of thorax & 6 & 2 & 4 & \\
\hline & Middle third of thorax & 32 & 7 & 25 & \\
\hline & Lower third of thorax & 21 & 10 & 11 & \\
\hline & Abdomen & 3 & 0 & 3 & \\
\hline \multirow[t]{2}{*}{ Tumor size, mm } & $<40$ & 25 & 6 & 19 & NS \\
\hline & $\geq 40$ & 39 & 15 & 24 & \\
\hline \multirow{3}{*}{$\begin{array}{l}\text { Histological } \\
\text { differentiation }\end{array}$} & Well-differentiated & 21 & 5 & 16 & NS \\
\hline & Moderately differentiated & 31 & 10 & 21 & \\
\hline & Poorly differentiated & 12 & 6 & 6 & \\
\hline \multirow[t]{2}{*}{ Vascular invasion } & Absent & 39 & 14 & 25 & NS \\
\hline & Present & 25 & 7 & 18 & \\
\hline \multirow{2}{*}{$\begin{array}{l}\text { Lymphatic } \\
\text { invasion }\end{array}$} & Absent & 20 & 6 & 14 & NS \\
\hline & Present & 44 & 15 & 29 & \\
\hline \multirow{2}{*}{$\begin{array}{l}\text { Lymph node } \\
\text { metastasis }\end{array}$} & Absent & 31 & 7 & 24 & NS \\
\hline & Present & 33 & 14 & 19 & \\
\hline \multirow{4}{*}{$\begin{array}{l}\text { Depth of tumor } \\
\text { invasion }\end{array}$} & pT1 & 32 & 6 & 26 & NS \\
\hline & pT2 & 8 & 3 & 5 & \\
\hline & pT3 & 23 & 11 & 12 & \\
\hline & pT4 & 1 & 1 & 0 & \\
\hline \multirow[t]{4}{*}{ Stage } & I & 24 & 5 & 19 & NS \\
\hline & $\mathrm{IIA}+\mathrm{B}$ & 19 & 5 & 14 & \\
\hline & III & 14 & 6 & 8 & \\
\hline & $\mathrm{IVA}+\mathrm{B}$ & 7 & 5 & 2 & \\
\hline
\end{tabular}

ESCC, esophageal squamous cell carcinoma.

Bedford, MA). Western blot analysis was carried out as described previously (26). Primary antibodies were used at dilution of 1:200 for anti-PBX2 and 1:1000 for anti-actin (Sigma). Density of each band was quantitated by Image $\mathbf{J}$ software.

WST-1 assay. To evaluate the effects of PBX2 on proliferation and apoptosis, cells $\left(1 \times 10^{4}\right)$ were cultured for $24 \mathrm{~h}$ with DMEM-10\% FBS or with serum-free DMEM. Premix WST-1 cell assay system (Takara Bio Inc, Kyoto, Japan) was used for evaluation of proliferative activity and viability of cells cultured with and without FBS, respectively. The absorbance at $450 \mathrm{~nm}$ was measured at 0 and 20 min after WST- 1 solution was added. The value at $20 \mathrm{~min}$ was divided with that at $0 \mathrm{~min}$, and the resultant was designated as proliferation or viability index. Experiments were carried out in triplicate, and the results were shown as mean \pm SD of three independent experiments.

Carboxyfluorescein diacetate succinimidyl ester (CFDA-SE) cell proliferation assay. The cells $\left(1 \times 10^{6} / \mathrm{ml}\right)$ were washed and suspended in $100 \mu \mathrm{l}$ PBS containing $0.1 \%$ BSA, and mixed with an equal volume of $50 \mu \mathrm{M}$ CFDA-SE (Invitrogen, final concentration $25 \mu \mathrm{M}$ ). After incubation at $37^{\circ} \mathrm{C}$ for $20 \mathrm{~min}, 1 \mathrm{ml}$ of DMEM medium with $10 \%$ FBS was added to stop the uptake of CFDA-SE dye. Labeled cells were cultured in DMEM-10\% FBS, and the rate of decrease in fluorescence intensity, i.e. the proliferative activity, was analyzed on a FACS Calibur flow cytometer (BD, Franklin Lakes, NJ) for 4 days. 
Table III. Univariate analysis of prognostic factors for DFS and OS in 94 pateints with GC.

\begin{tabular}{|c|c|c|c|c|c|c|}
\hline Clinicopathological features & Category & $\begin{array}{l}\text { No. of } \\
\text { patients }\end{array}$ & $\begin{array}{c}\text { 5-year DFS } \\
\text { rate }(\%)\end{array}$ & P-value & $\begin{array}{c}\text { 5-year OS } \\
\text { rate }(\%)\end{array}$ & P-value \\
\hline \multirow[t]{2}{*}{ PBX2 expression } & Low & 38 & 70.7 & $<0.01$ & 75.5 & $<0.01$ \\
\hline & High & 56 & 40.9 & & 49.5 & \\
\hline \multirow[t]{2}{*}{ Age } & $\leq 60$ & 36 & 69.2 & $<0.01$ & 77.0 & $<0.01$ \\
\hline & $>60$ & 58 & 42.6 & & 49.3 & \\
\hline \multirow[t]{2}{*}{$\operatorname{Sex}$} & Male & 65 & 44.0 & $<0.05$ & 51.3 & $<0.05$ \\
\hline & Female & 29 & 72.2 & & 79.3 & \\
\hline \multirow[t]{3}{*}{ Tumor location } & Upper third & 23 & 37.9 & NS & 42.1 & $<0.05^{\mathrm{a}}$ \\
\hline & Middle third & 23 & 56.5 & & 60.5 & $<0.01^{\mathrm{b}}$ \\
\hline & Lower third & 48 & 58.1 & & 68.4 & \\
\hline \multirow[t]{2}{*}{ Tumor size, $\mathrm{mm}$} & $<40$ & 68 & 61.4 & $<0.005$ & 66.9 & $<0.05$ \\
\hline & $\geq 40$ & 26 & 30.7 & & 41.9 & \\
\hline \multirow[t]{2}{*}{ Histological differentiation } & Undifferentiated & 55 & 41.8 & $<0.01$ & 50.7 & $<0.05$ \\
\hline & Differentiated & 39 & 68.7 & & 73.3 & \\
\hline \multirow[t]{2}{*}{ Vascular invasion } & Absent & 66 & 58.6 & $<0.05$ & 65.8 & $<0.05$ \\
\hline & Present & 28 & 38.9 & & 46.1 & \\
\hline \multirow[t]{2}{*}{ Lymphatic invasion } & Absent & 79 & 59.1 & $<0.0001$ & 65.1 & $<0.001$ \\
\hline & Present & 15 & 20.0 & & 33.3 & \\
\hline \multirow[t]{4}{*}{ Lymph node metastasis } & $\mathrm{pN}_{0}$ & 23 & 30.4 & $<0.001^{\mathrm{c}}$ & 47.8 & $<0.01^{\mathrm{d}}$ \\
\hline & $\mathrm{pN}_{1}$ & 52 & 11.5 & $<0.0001^{\mathrm{e}}$ & 26.9 & $<0.0001^{\mathrm{f}}$ \\
\hline & $\mathrm{pN}_{2}$ & 15 & 0.0 & & 6.6 & \\
\hline & $\mathrm{pN}_{3}$ & 4 & 0.0 & & 0.0 & \\
\hline \multirow[t]{4}{*}{ Depth of tumor invasion } & pT1 & 15 & 93.3 & $<0.001^{\mathrm{g}}$ & 100.0 & $<0.001^{\mathrm{h}}$ \\
\hline & pT2 & 17 & 88.2 & & 93.8 & \\
\hline & pT3 & 30 & 46.2 & & 59.1 & \\
\hline & pT4 & 32 & 21.8 & & 25.0 & \\
\hline \multirow[t]{2}{*}{ Chemotherapy } & Absent & 45 & 48.6 & NS & 50.3 & NS \\
\hline & Present & 49 & 56.6 & & 68.9 & \\
\hline
\end{tabular}

${ }^{\mathrm{a}} 1-2$ vs. 3 ; ${ }^{\mathrm{b}} 1$ vs. $2-3$; $^{\mathrm{c}}$ and ${ }^{\mathrm{d}} 1$ vs. $2-4{ }^{\mathrm{e}}$ and ${ }^{\mathrm{f}} 1-2$ vs. $3-4{ }^{\mathrm{g}}{ }^{\text {and }}{ }^{\mathrm{h}} 1-2$ vs. $3-4$. DFS, desease-free survival; OS, overall survival; GC, gastric cancer; vs., versus.

Staining of apoptotic cells with annexin $V$. Apoptotic cells were stained with annexin V-FITC using the Apoptosis Detection kit (MBL, Nagoya, Japan), and subsequently analyzed by fluorescent microscope (Biozero, BZ-8000, Keyence, Japan).

Matrigel invasion assay. Invasion of tumor cells into the Matrigel was examined with BD BioCoat Matrigel invasion chamber (BD Biosciences, San Jose, CA). Briefly, cells were seeded in DMEM without FBS in the Matrigel invasion chamber, and cultured in DMEM-10\% FBS for $60 \mathrm{~h}$. Invading cells were stained with Diff-quick staining kit (Siemens, Munich, Germany). Number of invading cells was counted at five microscopic fields per well at a magnification of $\mathrm{x} 100$, and the extent of invasion was expressed as the average number of cells per $\mathrm{mm}^{2}$.
In vitro colony formation assay. A total of $1 \times 10^{3}$ cells were plated in $60 \mathrm{~mm}$ culture dishes and kept for 14 days in a medium with $15 \%$ FBS-containing methylcellulose, and the number of formed colonies was counted.

Real-time quantitative polymerase chain reaction ( $R T-Q P C R)$. Total RNA was extracted from PBX2 knocked down cell lines of MNK-45 and KYSE70 using RNeasy RNA extraction kit (Qiagen, Valencia, CA) according to the manufacturer's protocol, and cDNA was synthesized using oligo (dT) primers and SuperScript III reverse transcriptase (Invitrogen). RTQPCR was used to quantify mRNA expression of PBX2 and Bcl-2 using an ABI PRISM 7700 instrument (Applied Biosystems, Foster City, CA). GAPDH was used as a reference for gene amplification (Applied Biosystems). 
Table IV. Multivariate analysis of prognostic factors for DFS and OS in 94 patients with GC.

\begin{tabular}{|c|c|c|c|c|c|}
\hline Factor & Category & Relative risk & $95 \%$ Confidence interval & $\chi^{2}$ value & P-value \\
\hline \multicolumn{6}{|l|}{ Overall survival } \\
\hline PBX2 expression & $\begin{array}{l}\text { Low } \\
\text { High }\end{array}$ & 1.683 & $1.122-2.625$ & 6.414 & $<0.05$ \\
\hline Age & $\begin{array}{l}\leq 60 \\
>60\end{array}$ & 1.032 & $0.678-1.640$ & 0.02 & 0.886 \\
\hline Sex & $\begin{array}{l}\text { Male } \\
\text { Female }\end{array}$ & 1.094 & $0.695-1.858$ & 0.135 & 0.713 \\
\hline Tumor location & $\begin{array}{l}\text { Upper } \\
\text { Middle+Lower }\end{array}$ & 0.664 & $0.456-0.990$ & 4.023 & $<0.05$ \\
\hline Tumor size, mm & $\begin{array}{l}<40 \\
\geq 40\end{array}$ & 1.001 & $0.686-1.445$ & 0.00001 & 0.995 \\
\hline $\begin{array}{l}\text { Histological } \\
\text { differentiation }\end{array}$ & $\begin{array}{l}\text { Undifferentiated } \\
\text { Differentiated }\end{array}$ & 1.844 & $1.222-2.871$ & 8.647 & $<0.005$ \\
\hline Vascular invasion & $\begin{array}{l}\text { Absent } \\
\text { Present }\end{array}$ & 1.149 & $0.797-1.640$ & 0.573 & 0.449 \\
\hline Lymphatic invasion & $\begin{array}{l}\text { Absent } \\
\text { Present }\end{array}$ & 1.108 & $0.729-1.627$ & 0.25 & 0.617 \\
\hline Lymph node metastasis & $\begin{array}{l}\mathrm{pN}_{0}+\mathrm{pN}_{1} \\
\mathrm{pN}_{2}+\mathrm{pN}_{3}\end{array}$ & 2.129 & $1.406-3.221$ & 12.381 & $<0.0005$ \\
\hline Depth of tumor invasion & $\begin{array}{l}\mathrm{pT}_{3}+\mathrm{pT}_{4} \\
\mathrm{pT}_{1}+\mathrm{pT}_{2}\end{array}$ & 4.835 & $2.088-21.007$ & 19.579 & $<0.0005$ \\
\hline \multicolumn{6}{|l|}{ Disease-free survival } \\
\hline PBX2 expression & $\begin{array}{l}\text { Low } \\
\text { High }\end{array}$ & 1.84 & $1.274-2.761$ & 11.091 & $<0.005$ \\
\hline Age & $\begin{array}{l}\leq 60 \\
>60\end{array}$ & 1.056 & $0.734-1.564$ & 0.082 & 0.775 \\
\hline Sex & $\begin{array}{l}\text { Male } \\
\text { Female }\end{array}$ & 1.04 & $0.701-1.62$ & 0.034 & 0.854 \\
\hline Tumor size, mm & $\begin{array}{l}<40 \\
\geq 40\end{array}$ & 1.02 & $0.719-1.438$ & 0.013 & 0.908 \\
\hline $\begin{array}{l}\text { Histological } \\
\text { differentiation }\end{array}$ & $\begin{array}{l}\text { Undifferentiated } \\
\text { Differentiated }\end{array}$ & 1.673 & $1.174-2.450$ & 8.261 & $<0.005$ \\
\hline Vascular invasion & $\begin{array}{l}\text { Absent } \\
\text { Present }\end{array}$ & 1.027 & $0.728-1.432$ & 0.024 & 0.877 \\
\hline Lymphatic invasion & $\begin{array}{l}\text { Absent } \\
\text { Present }\end{array}$ & 1.256 & $0.860-1.790$ & 1.437 & 0.231 \\
\hline Lymph node metastasis & $\begin{array}{l}\mathrm{pN}_{0}+\mathrm{pN}_{1} \\
\mathrm{pN}_{2}+\mathrm{pN}_{3}\end{array}$ & 1.948 & $1.310-2.866$ & 10.431 & $<0.005$ \\
\hline Depth of tumor invasion & $\begin{array}{l}\mathrm{pT}_{3}+\mathrm{pT}_{4} \\
\mathrm{pT}_{1}+\mathrm{pT}_{2}\end{array}$ & 2.979 & $1.666-6.374$ & 16.208 & $<0.0005$ \\
\hline
\end{tabular}


Table V. Univariate analysis of prognostic factors for DFS and OS in 64 patients with ESCC.

\begin{tabular}{|c|c|c|c|c|c|c|}
\hline Factors & Category & $\begin{array}{l}\text { No. of } \\
\text { patients }\end{array}$ & $\begin{array}{c}\text { 5-year DFS } \\
\text { rate }(\%)\end{array}$ & P-value & $\begin{array}{c}\text { 5-year OS } \\
\text { rate }(\%)\end{array}$ & P-value \\
\hline \multirow[t]{2}{*}{ PBX2 expression } & High & 21 & 56.1 & \multirow[t]{2}{*}{$<0.01$} & 56.4 & \multirow[t]{2}{*}{$<0.01$} \\
\hline & Low & 43 & 85.0 & & 86.3 & \\
\hline \multirow[t]{2}{*}{ Gender } & Male & 59 & 76.5 & \multirow[t]{2}{*}{ NS } & 77.6 & \multirow[t]{2}{*}{ NS } \\
\hline & Female & 5 & 40.0 & & 50.0 & \\
\hline \multirow[t]{5}{*}{ Tumor location } & Cervix & 2 & 0.0 & \multirow[t]{5}{*}{ NS } & 0.0 & \multirow[t]{5}{*}{ NS } \\
\hline & Upper third of thorax & 6 & 33.3 & & 50.0 & \\
\hline & Middle third of thorax & 32 & 37.5 & & 37.5 & \\
\hline & Lower third of thorax & 21 & 28.6 & & 33.3 & \\
\hline & Abdomen & 3 & 0.0 & & 0.0 & \\
\hline \multirow[t]{2}{*}{ Tumor size (mm) } & $<40$ & 25 & 75.8 & \multirow[t]{2}{*}{ NS } & 78.2 & \multirow[t]{2}{*}{ NS } \\
\hline & $\geq 40$ & 39 & 74.8 & & 73.9 & \\
\hline \multirow{3}{*}{$\begin{array}{l}\text { Histologic } \\
\text { differentiation }\end{array}$} & Well-differentiated & 21 & 33.3 & \multirow[t]{3}{*}{$<0.05^{\mathrm{a}}$} & 38.1 & \multirow[t]{3}{*}{$<0.05^{b}$} \\
\hline & Moderately differentiated & 31 & 32.3 & & 35.5 & \\
\hline & Poorly differentiated & 12 & 25.0 & & 25.0 & \\
\hline \multirow[t]{2}{*}{ Vascular invasion } & Absent & 39 & 86.9 & \multirow[t]{2}{*}{$<0.005$} & 85.5 & \multirow[t]{2}{*}{$<0.05$} \\
\hline & Present & 25 & 55.4 & & 59.7 & \\
\hline \multirow[t]{2}{*}{ Lymphatic invasion } & Absent & 20 & 65.7 & \multirow[t]{2}{*}{$<0.05$} & 69.5 & \multirow[t]{2}{*}{ NS } \\
\hline & Present & 44 & 31.8 & & 88.5 & \\
\hline \multirow[t]{2}{*}{ Lymph node metastasis } & Absent & 31 & 86.4 & \multirow[t]{2}{*}{$<0.005$} & 88.6 & \multirow[t]{2}{*}{$<0.005$} \\
\hline & Present & 33 & 42.9 & & 36.4 & \\
\hline \multirow[t]{4}{*}{ Depth of tumor invasion } & pT1 & 32 & 43.8 & \multirow{4}{*}{$\begin{array}{l}<0.0001^{\mathrm{c}} \\
<0.001^{\mathrm{e}}\end{array}$} & 43.8 & \multirow{4}{*}{$\begin{array}{l}<0.01^{\mathrm{d}} \\
<0.005^{\mathrm{f}}\end{array}$} \\
\hline & pT2 & 8 & 25.0 & & 37.5 & \\
\hline & pT3 & 23 & 17.4 & & 21.7 & \\
\hline & pT4 & 1 & 0.0 & & 0.0 & \\
\hline \multirow[t]{4}{*}{ Stage } & I & 24 & 45.8 & \multirow[t]{4}{*}{$<0.001^{\mathrm{g}}$} & 45.8 & \multirow[t]{4}{*}{ NS } \\
\hline & $\mathrm{IIA}+\mathrm{B}$ & 19 & 21.1 & & 21.1 & \\
\hline & III & 14 & 14.3 & & 14.3 & \\
\hline & $\mathrm{IVA}+\mathrm{B}$ & 7 & 42.8 & & 57.1 & \\
\hline
\end{tabular}

${ }^{\mathrm{a}}$ and ${ }^{\mathrm{b}} 1-2$ vs. $3 ;{ }^{\mathrm{c}}$ and ${ }^{\mathrm{d}} 1$ vs. $2-4 ;{ }^{\mathrm{e}}$ and ${ }^{\mathrm{f}} 1-2$ vs. $3 ;{ }^{\mathrm{g}} 1-2$ vs. $3-4$. DFS, desease-free survival; OS, overall survival; ESCC, esophageal squamous cell carcinoma; vs., versus.

Mice and xenograft transplantation. Six to 8-week-old female non-obese diabetic (NOD)/Scid mice were purchased from Charles River Laboratories Japan (Kanagawa, Japan) and kept under specific pathogen-free conditions. Before xenotransplantation, the mice were deeply anesthetized. All animal experiments were performed according to the guideline of Osaka University Animal Center, and approved by the institutional review board of committee of animal experiments (no. 753). For xenograft transplantation, KYSE70 cells $\left(1 \times 10^{4}\right)$ of PBX2 knocked down (KD1) and control (KC) were suspended in $0.2 \mathrm{ml}$ of Matrigel (BD Biosciences), and were injected subcutaneously into NOD/Scid mice, respectively.
The tumor volume was measured using the following formula: (width) $)^{2} \mathrm{x}$ (length)/2 according to the report by Meyer-Siegler et al (27).

Statistical analysis. Statistical analysis for experimental studies was carried out using Student's t-tests. The values are shown as the mean \pm SE of at least three experiments. Statistical analyses for clinical samples were performed using JMP software (SAS Institute Inc., Cary, NC). Overall survival (OS) and disease-free survival (DFS) were measured from the date of surgery to death by any causes and to disease recurrence or occurrence of distant metastases, respectively. 
Table VI. Multivariate analysis of prognostic factors for DFS and OS in 64 patients with ESCC.

\begin{tabular}{|c|c|c|c|c|c|}
\hline Factors & Category & Relative risk & 95\% Confidence interval & $\chi^{2}$ value & P-value \\
\hline \multicolumn{6}{|l|}{ Overall survival } \\
\hline PBX2 expression & $\begin{array}{l}\text { High } \\
\text { Low }\end{array}$ & 1.966 & $1.087-3.703$ & 5.005 & $<0.05$ \\
\hline Histologic differentiation & $\begin{array}{l}\text { Well+moderately } \\
\text { Poorly }\end{array}$ & 2.307 & $1.166-4.569$ & 5.645 & $<0.05$ \\
\hline Vascular invasion & $\begin{array}{l}\text { Absent } \\
\text { Present }\end{array}$ & 2.146 & $1.210-3.985$ & 6.776 & $<0.01$ \\
\hline Depth of tumor invasion & $\begin{array}{l}\text { pT1 } \\
\text { pT2-4 }\end{array}$ & 1.664 & $0.875-3.669$ & 2.347 & 0.126 \\
\hline \multicolumn{6}{|l|}{ Disease-free survival } \\
\hline PBX2 expression & $\begin{array}{l}\text { High } \\
\text { Low }\end{array}$ & 2.052 & $1.003-4.553$ & 2.052 & $<0.05$ \\
\hline Histologic differentiation & $\begin{array}{l}\text { Well+moderately } \\
\text { Poorly }\end{array}$ & 0.920 & $0.481-1.783$ & 0.065 & 0.800 \\
\hline Vascular invasion & $\begin{array}{l}\text { Absent } \\
\text { Present }\end{array}$ & 1.981 & $1.008-1.149$ & 3.930 & $<0.05$ \\
\hline Lymphatic invasion & $\begin{array}{l}\text { Absent } \\
\text { Present }\end{array}$ & 1.465 & $0.594-6.532$ & 0.552 & 0.457 \\
\hline Depth of tumor invasion & $\begin{array}{l}\text { pT1 } \\
\text { pT2-4 }\end{array}$ & 2.680 & $0.984-12.149$ & 3.710 & 0.054 \\
\hline Stage & $\begin{array}{l}\mathrm{I}+\mathrm{II} \\
\mathrm{III}+\mathrm{IV}\end{array}$ & 0.890 & $0.463-1.811$ & 0.117 & 0.732 \\
\hline
\end{tabular}

DFS, desease-free survival; OS, overall survival; ESCC, esophageal squamous cell carcinoma; vs., versus.

Follow-up period for the survivors of GC and ESCC ranged from 1.8 to 94 (median 39.5) months and from 1.6 to 94 (median 46.4) months, respectively. The $\chi^{2}$ and Fisher's exact tests were used to analyze the correlation between PBX2 expression determined by immunohistochemistry and clinicopathologic features of GC and ESCC. Kaplan-Meier method and log-rank tests were used to evaluate the survival rates and differences in survival curves. In the multivariate analysis, independent prognostic factors for survival of patients were determined by using Cox regression hazards model. $\mathrm{P}<0.05$ were considered to be statistically significant.

\section{Results}

Clinicopathological features. The 94 cases of GC histologically consisted of 18 cases with well-differentiated, 19 moderately-differentiated, 48 poorly-differentiated adenocarcinoma, four signet ring cell carcinoma, three mucinous carcinoma, and one each of papillary carcinoma and medullary carcinoma. Cases with poorly-differentiated, signet ring cell, and mucinous carcinomas were unified and categorized as undifferentiated carcinoma. Other cases were unified as differentiated ones. Tumor cells invaded into mucosa or submucosa $\left(\mathrm{pT}_{1}\right)$ in 15 cases, muscularis propria or subserosa $\left(\mathrm{pT}_{2}\right)$ in 17 , serosa $\left(\mathrm{pT}_{3}\right)$ in 30 , and adjacent organs $\left(\mathrm{pT}_{4}\right)$ in 32 . Seventy-two cases were node-negative and 23 node positive. In GC samples, $56(59.6 \%)$ and $38(40.4 \%)$ cases showed high and low PBX2 expression, respectively (Fig. 1A and B). None of the clinicopathological features correlated with PBX2 expression level (Table I).

In the 64 cases of ESCC, 21 had well differentiated, 31 moderately differentiated, and 12 poorly differentiated squamous cell carcinomas. Tumor cells invaded into the mucosa or submucosa $\left(\mathrm{pT}_{1}\right)$ in 32 cases, muscularis propria or subadventitia $\left(\mathrm{pT}_{2}\right)$ in 8 , adventitia $\left(\mathrm{pT}_{3}\right)$ in 23 , and adjacent organs $\left(\mathrm{pT}_{4}\right)$ in one. Twenty-one $(32.8 \%)$ and $43(67.2 \%)$ cases showed a low and high PBX2 expression, respectively (Fig. 1C and D). None of the clinicopathological features correlated with PBX2 expression level (Table II).

Univariate and multivariate analysis for prognostic factors of GC or ESCC. Five-year DFS and OS rate of the GC patients was 53.1 and $60.6 \%$, respectively. Tumor recurrence was observed in 44 patients; peritoneum in eight, lymph nodes in nine, liver in six, lung in four, colon in four, esophagus in two, liver in three, bone in one, and multiple visceral meta- 

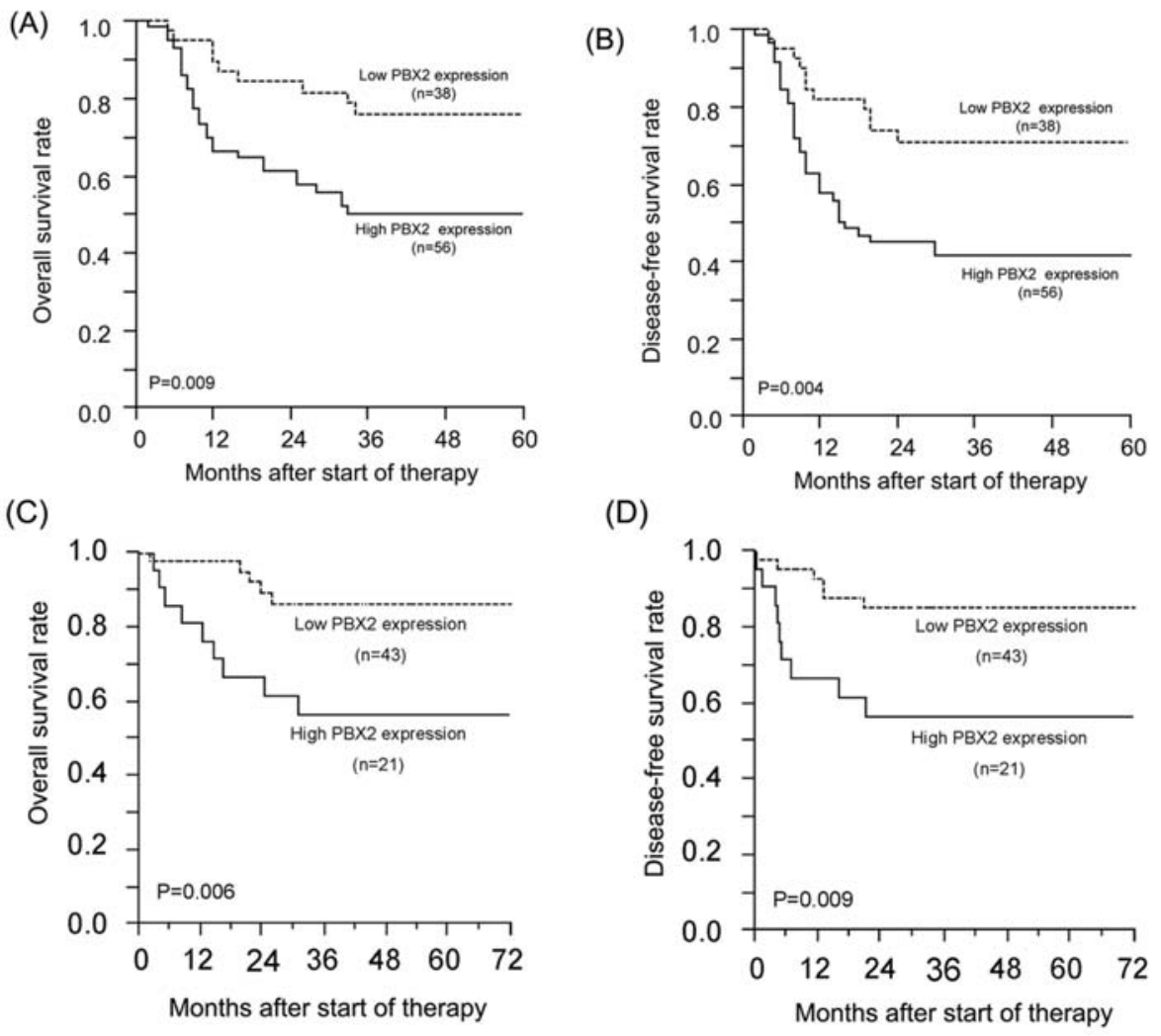

Figure 2. Kaplan-Meier plots of OS and DFS. Kaplan-Meier plots of OS and DFS of patients with high and low expression in GC (A and B) and ESCC (C and D).

stases in seven. Twenty-two patients died due to tumor. The univariate analysis revealed that gender and age of patients, size of tumor, tumor location, histologic differentiation, tumor invasion beyond propria muscle, vascular and lymphatic invasion, lymph node metastasis, and PBX2 expression were significant factors for both DFS and OS (Table III). Patients with high PBX2 expression showed worse OS and DSF than those with low expression (Fig. 2A and B). Multivariate analysis with factors proven to be significant in the univariate analysis revealed that PBX2 expression, depth of tumor invasion, histological differentiation, and lymph node metastasis were independent prognostic factors for both DFS and OS. Tumor location is an independent prognosticator for OS but not for DFS (Table IV).

As for ESCC patients, the 5-year DFS and OS rate was 57.1 and $61.1 \%$, respectively. ESCC patients with low PBX2 expression had better OS and DFS than those with high expression (Fig. 2C and D). Univariate analysis revealed that histologic differentiation, depth of tumor invasion, vascular invasion, and lymph node metastasis were significant factors for both DFS and OS. Lymphatic invasion and stage significantly affected DFS but not OS (Table V). Multivariate analysis with factors proven to be significant in the univariate analysis revealed that PBX2 expression level in tumor cells and vascular invasion were independent prognostic factors for both DFS and OS, and histologic differentiation for OS (Table VI).

Knocked-down expression of PBX2 in GC and ESCC cell lines. To examine the effects of PBX2 on GC and ESCC cells, expression of $\mathrm{PBX} 2$ was knocked-down in $\mathrm{GC}$ cell line

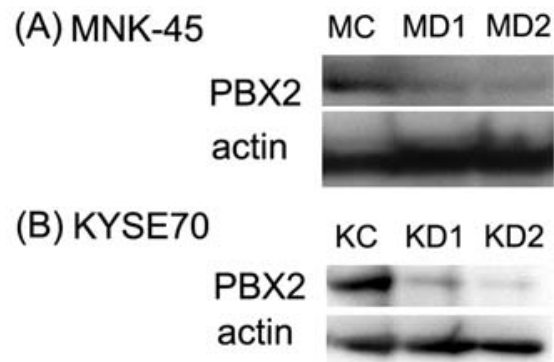

Figure 3. Expression of PBX2 in MNK-45 and KYSE70 cells examined by WB. (A) Amount of PBX2 protein in the control (MC) and si-RNA knocked-down (MD1 and MD2) MNK-45 cells. (B) Amount of PBX2 protein in the control (KC) and si-RNA knocked-down (KD1 and KD2) KYSE70 cells.

MNK-45 and ESCC cell line KYSE70. Four stable clones of independent PBX2 knocked-down cells were obtained from MNK-45 (MD1 and MD2) and KYSE70 (KD1 and KD2), respectively. When compared to control clones from the MNK-45 (MC) and KYSE70 (KC), expression amount of PBX2 protein in the knocked-down clones reduced (Fig. 3A and B). Effect of PBX2 on the cell proliferation was subsequently examined with WST-1 assay. No remarkable changes of proliferative activity were found between the control and knocked-down cells (Fig. 4A). For further characterization of cell proliferation, the control and knocked-down cells were labeled with CFDA-SE fluorescent dye, and time-dependent diminishment of fluorescent intensity was analyzed. When a single cell is divided into two, the original fluorescent intensity 

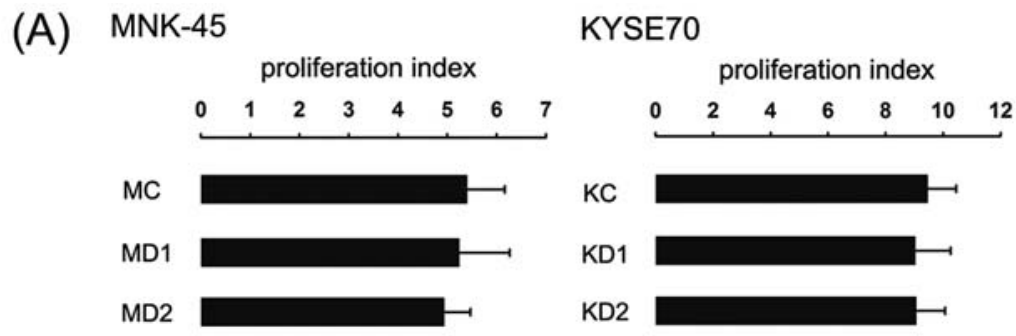

(B)

\section{MNK-45}

MC

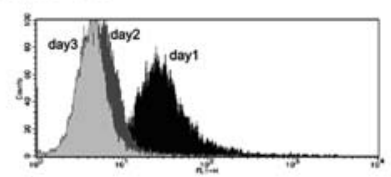

KYSE70

MD1

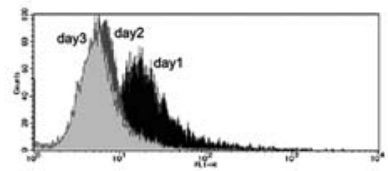

$\mathrm{KC}$

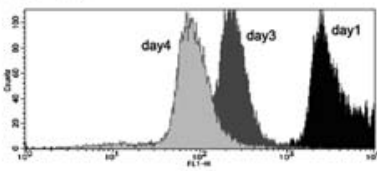

MD2

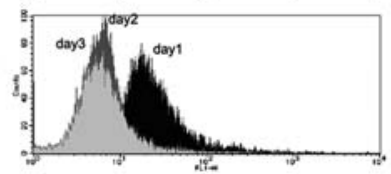

(C)
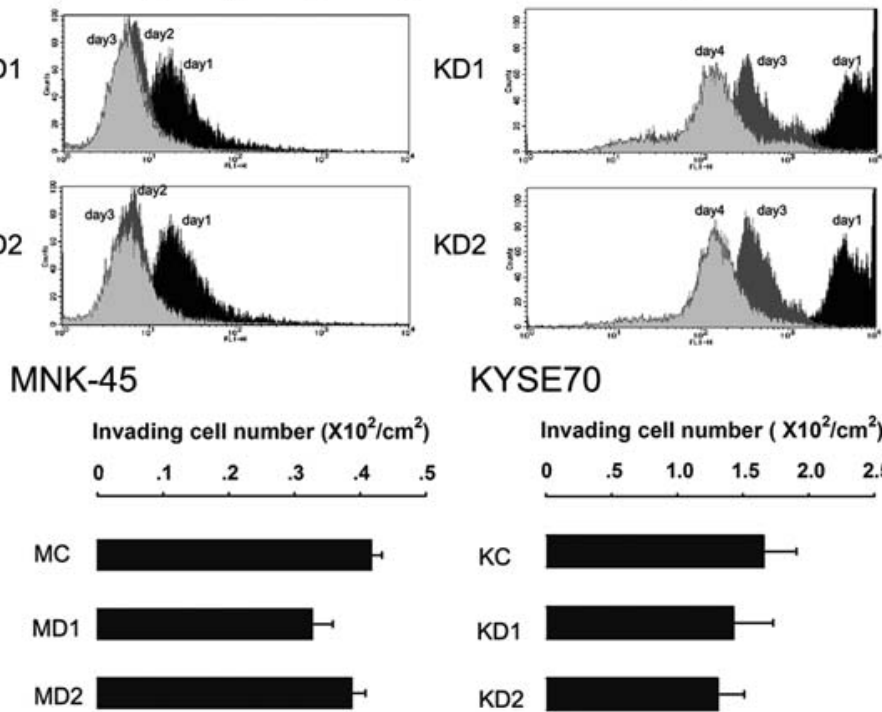

KD2

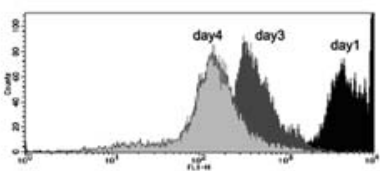

KYSE70

Invading cell number $\left(X_{10}^{2} / \mathrm{cm}^{2}\right)$

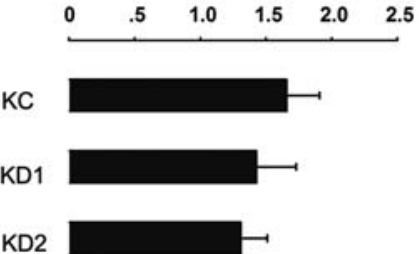

Figure 4. Effect of PBX2 expression on the proliferative and invasion activity in MNK-45 and KYSE70 cells. (A) Comparison of proliferation of PBX2 knocked down cells to that of control cells. (B) Cell proliferative activity as revealed by CFDA-SE in MNK-45 and KYSE70 cells for 3 days. (C) Comparison of invasion activity of PBX2 knocked down cells to that of control cells.

decreases to one half, showing that the proliferative ability correlated with the diminishing rate of fluorescent intensity. No obvious differences in diminishing rate were detected between the control and knocked-down cells (Fig. 4B). These findings indicate that $\mathrm{PBX} 2$ did not show any effect on proliferation rate of GC and ESCC cell lines. Next, the effect of PBX2 on invasion ability was examined with Matrigel invasion assay: number of invading cells in knocked-down cells reduced, but the difference between the control and knocked-down cells was not significant (Fig. 4C).

Effect of PBX2 on colony formation and tumorigenic abilities. The effects of PBX2 on in vitro colony formation and in vivo tumorigenic activities were evaluated. As compared to the control cells, the PBX2 knocked-down cells formed lower number of colonies in both GC and ESCC cell lines (Fig. 5A). Next, $1 \times 10^{6} \mathrm{KC}$ and $\mathrm{KD} 1$ cells were injected into the left and right flank of $2 \mathrm{NOD} /$ Scid mice. After 5 weeks, tumors were resected. The sizes of tumors derived from PBX2 knockeddown cells were significantly smaller than those of the control cells (Fig. 5B). Histologically, both tumors derived from the control and knocked-down cells showed squamoid pattern of proliferation (Fig. 5C).
Vulnerability to apoptosis in PBX2-knocked down cells. Resistance to apoptotic stimuli was analyzed in PBX2 knocked-down cells. Under serum-depleted condition, viability of PBX2 knocked-down cells decreased more than that of control cells (Fig. 6A). Content of early apoptotic cells was examined with annexin V staining. Amount of early apoptotic cells was significantly higher in PBX2 knocked-down cells than in control cells of GC and ESCC (Fig. 6B and C).

To examine why the content of apoptotic cells increased by PBX2 knocked-down, expression level of Bcl-2, which plays an important role for elongation of programmed cell death, was examined in the GC and ESCC cell lines. The real-time RT-PCR revealed that the amount of Bcl-2 mRNA in PBX2 knocked-down cells was significantly decreased compared to the control cells in both GC and ESCC cell lines (Fig. 7A and B).

\section{Discussion}

The patient characteristics, such as gender, age, stage, histologic type, and 5-year survival rate in the present series were similar to those in the previous reports from China, Japan, and other countries where GC and ESCC are common 
(A)

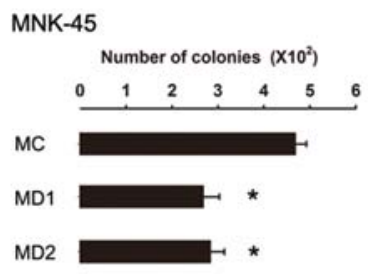

(B)

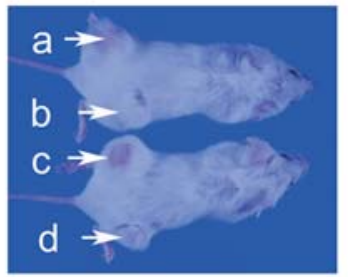

(C)
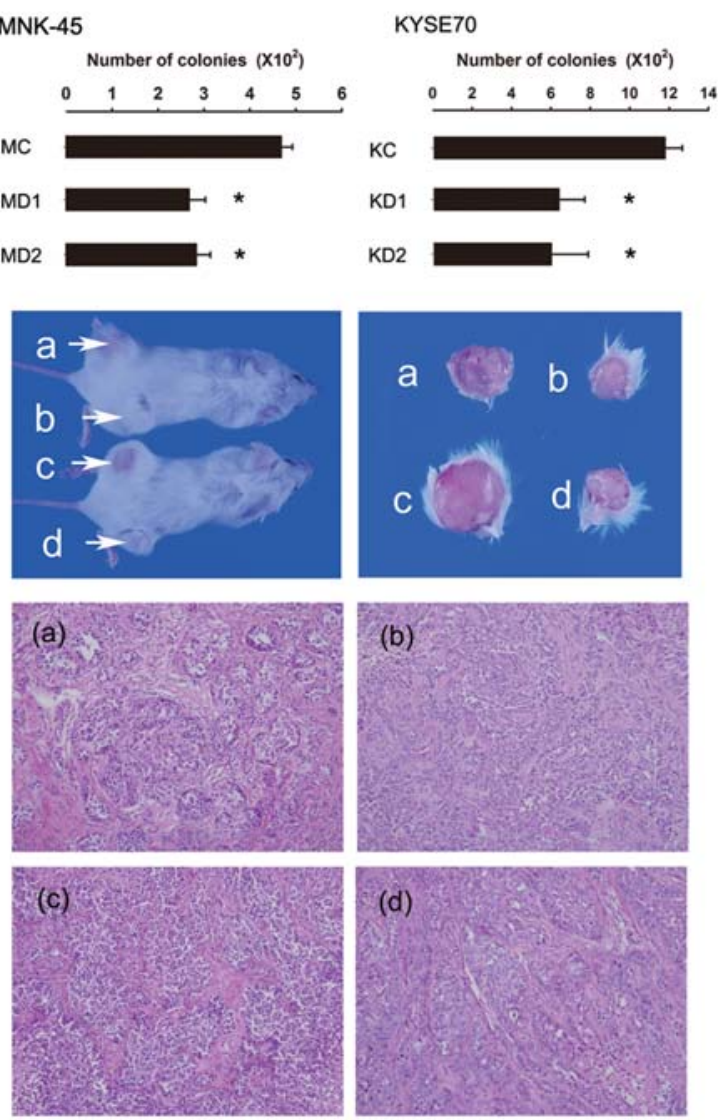

Figure 5. Effects of $\mathrm{PBX} 2$ on in vitro colony formation and in vivo tumorigenic activities. (A) Number of colonies was compare between the control and the PBX2 knocked-down cells (B) Tumors in NOD/Scid mice at the injection sites of the control (a and $c$ ) and the knocked-down (b and d) KYSE70 cells. (C) Histology of tumor derived from the control (a and c) and the knocked-down (b and d) cells. $\mathrm{x} 400$ The values in (A) represent the mean \pm SE of three experiments. ${ }^{*} \mathrm{p}<0.01$ by the Student's t-test.
(A)

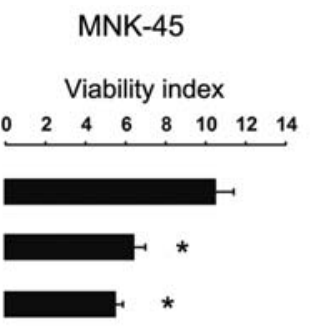

(B)
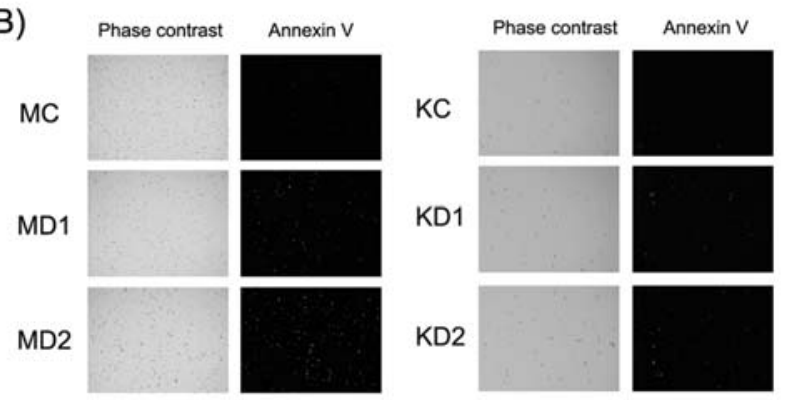

(C)

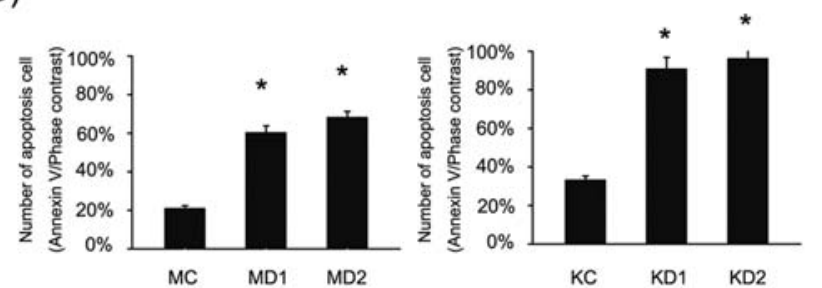

Figure 6. Effects of PBX2 expression on apoptosis of MNK-45 and KYSE70 cells. (A) Comparison of viability of PBX2 knocked-down cells to that of control cells. (B) Cells in apoptosis were compared between PBX2 knockdown and control cells. (C) Comparison of apoptotic cell proportion of PBX2 knocked-down cells to that of control cells. The values in (C) represent the mean \pm SE of three experiments. ${ }^{*} \mathrm{p}<0.001$.

\section{(A) MNK-45}

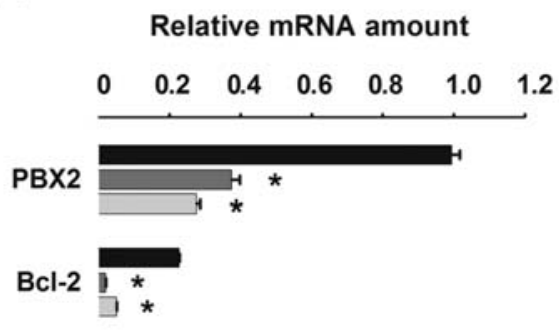

(B) KYSE70

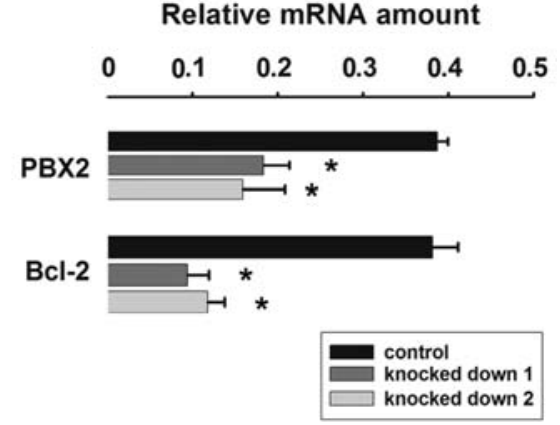

Figure 7. Expression of Bcl-2 gene in PBX2 knocked-down MNK-45 (A) and KYSE70 (B) cells. Relative amounts of PBX2 and Bcl-2 mRNA were shown. Amounts of Bcl-2 mRNA significantly reduced when PBX2 expression levels decreased. * $\mathrm{P}<0.05$ by Student's t-test.

(28-30). In addition, the present study also showed that clinicopathological variables, such as tumor invasion, occurrence of metastasis, vascular and lymphatic invasion, and serosal invasion were significant factors for prediction of long-term survival of GC or ESCC patients as reported previously $(28,31,32)$. These findings indicate that the results obtained from the present cases are commonly applicable. Our previous study showed that the high expression of PBX2 in the tumor cells was an indicator of poor prognosis in NSCLC (24). The present study also showed the prognostic significance of PBX2 expression level for both GC and ESCC, but the effect of PBX2 expression on survival was more profound, PBX2 expression was an independent prognostic indicator for GC and ESCC.

$\mathrm{PBX} 2$ protein, a main member of $\mathrm{PBX}$ family, is ubiquitously expressed in various tissues with different expression 
levels by tissues. PBX2 works as a cofactor with other proteins, such as HOX, and forms dimeric complexes with increasing DNA binding affinity and specificity (33). HOX genes are essential for all aspects of mammalian growth and differentiation. Several studies indicated that PBX proteins function in vivo as regulators of differentiation and function in concert with HOX proteins in development (34). These protein complexes, including PBX2 protein, regulate the expression of number of genes, thus induce the execution of various cellular functions, such as anti-apoptosis, and inhibition of cell differentiation and proliferation (35).

It has been shown that deregulated expression of HOX genes is involved in carcinogenesis, i.e., the high expression of HOX correlated with poor prognosis of patients in acute myeloid leukemia and lymphoid malignancies $(13,36,37)$. Several studies showed significantly higher level of HOX expression in cancer cells compared to normal mucosa in ESCC and GC $(6,38)$. The synergistic effects of HOX proteins and $\mathrm{PBX} 2$ protein findings explain why $\mathrm{PBX} 2$ expression correlates with poor prognosis of GC and ESCC patients. PBX2 could play a significant role in tumor development.

To evaluate functional sequels induced by PBX2 expression, PBX2 siRNA plamids were constructed and transfected into the GC and ESCC cell lines. The knockeddown expression of $\mathrm{PBX} 2$ resulted in decrease of in vitro colony formation and in vivo tumorigenic activities. Correlation of PBX2 expression level with tumorigenic activity of the cultured cells was consistent with findings found in clinical cases that tumor recurrence rate was higher in the cases with high PBX2 expression than those with low expression. PBX2 expression level was not correlated with tumor size, frequencies of lymph node metastasis and vascular and lymphatic invasions in patients of GC and ESCC. These findings suggest that PBX2 might not promote tumor proliferation and lymphovascular invasion.

The knocked-down expression of PBX2 did not change proliferation and invasion capacities of the cultured cells, but increase of apoptosis rate and the decrease of Bcl-2 expression were found. These findings suggested that PBX2 expression accelerated tumor growth through escape from apoptosis. Supporting evidence for this has been reported. Potts et al reported that HOX protein ensures cell survival by inhibition of apoptosis, thus promotes leukemia development (39). Decreased activity of HOX proteins by interfering their binding to the PBX co-factor causes apoptosis of cancer cell lines in vitro and reduces the growth in vivo $(15,40)$.

In conclusion, the present study clearly demonstrated that the increased expression of PBX2 in GC and ESCC is a sign for poor prognosis and $\mathrm{PBX} 2$ is an independent prognosticator for GC and ESCC patients. PBX2 might promote tumor growth through escape of cancer cells from apoptosis.

\section{Acknowledgements}

This study was supported in part by the International Collaborative Project of National Natural Science Foundation of China (no. 30801382) and by a grant from the Ministry of Education, Culture, Sports, Science and Technology (20014010, 20014012, and 20590364), Japan.

\section{References}

1. McGinnis W and Krumlauf R: Homeobox genes and axial patterning. Cell 68: 283-302, 1992.

2. Phelan ML, Rambaldi I and Featherstone MS: Cooperative interactions between Hox and $\mathrm{Pbx}$ proteins mediated by a conserved peptide motif. Mol Cell Biol 15: 3989-3997, 1995.

3. Hassan NM, Hamada J, Murai T, et al: Aberrant expression of HOX genes in oral dysplasia and squamous cell carcinoma tissues. Oncol Res 16: 217-224, 2006.

4. Hamada Ji, Omatsu T, Okada F, et al: Overexpression of homeobox gene HOXD3 induces coordinate expression of metastasis-related genes in human lung cancer cells. Int J Cancer 93: 516-525, 2001.

5. Naora H, Yang YQ, Montz FJ, et al: A serologically identified tumor antigen encoded by a homeobox gene promotes growth of ovarian epithelial cells. Proc Natl Acad Sci USA 98: 4060-4065, 2001.

6. Takahashi O, Hamada J, Abe M, et al: Dysregulated expression of HOX and ParaHOX genes in human esophageal squamous cell carcinoma. Oncol Rep 17: 753-760, 2007.

7. Rossi Degl'Innocenti D, Castiglione F, Buccoliero AM, et al: Quantitative expression of the homeobox and integrin genes in human gastric carcinoma. Int J Mol Med 20: 621-629, 2007.

8. Shimoda M, Takahashi M, Yoshimoto T, et al: A homeobox protein, prox 1, is involved in the differentiation, proliferation, and prognosis in hepatocellular carcinoma. Clin Cancer Res 12: 6005-6011, 2006.

9. Miller GJ, Miller HL, van Bokhoven A, et al: Aberrant HOXC expression accompanies the malignant phenotype in human prostate. Cancer Res 63: 5879-5888, 2003.

10. Cantile M, Cindolo L, Napodano G, et al: Hyperexpression of locus $\mathrm{C}$ genes in the HOX network is strongly associated in vivo with human bladder transitional cell carcinomas. Oncogene 22: 6462-6468, 2003.

11. Li H, Huang CJ and Choo KB: Expression of homeobox genes in cervical cancer. Gynecol Oncol 84: 216-221, 2002.

12. Rozovskaia T, Feinstein E, Mor O, et al: Upregulation of Meis 1 and HoxA9 in acute lymphocytic leukemias with the $\mathrm{t}(4: 11)$ abnormality. Oncogene 20: 874-878, 2001.

13. Eklund EA: The role of HOX genes in malignant myeloid disease. Curr Opin Hematol 14: 85-89, 2007.

14. Golub TR, Slonim DK, Tamayo P, et al: Molecular classification of cancer: class discovery and class prediction by gene expression monitoring. Science 286: 531-537, 1999.

15. Plowright L, Harrington KJ, Pandha HS, et al: HOX transcription factors are potential therapeutic targets in non-smallcell lung cancer (targeting $\mathrm{HOX}$ genes in lung cancer). Br J Cancer 100: 470-475, 2009.

16. Nakamura T, Jenkins NA and Copeland NG: Identification of a new family of Pbx-related homeobox genes. Oncogene 13: 2235-2242, 1996.

17. Aulisa L, Forraz N, McGuckin C, et al: Inhibition of cancer cell proliferation by designed peptide amphiphiles. Acta Biomater 5: 842-853, 2009.

18. Kamangar F, Dores GM and Anderson WF: Patterns of cancer incidence, mortality, and prevalence across five continents: defining priorities to reduce cancer disparities in different geographic regions of the world. J Clin Oncol 24: 2137-2150, 2006.

19. Enzinger PC and Mayer RJ: Esophageal cancer. N Engl J Med 349: 2241-2252, 2003.

20. Daly JM, Fry WA, Little AG, et al: Esophageal cancer: results of an American College of Surgeons patient care evaluation study. J Am Coll Surg 190: 562-572, 2000.

21. Buskens CJ, Ten Kate FJ, Obertop H, et al: Analysis of micrometastatic disease in histologically negative lymph nodes of patients with adenocarcinoma of the distal esophagus or gastric cardia. Dis Esophagus 21: 488-495, 2007.

22. Lambert R, Guilloux A, Oshima A, et al: Incidence and mortality from stomach cancer in Japan, Slovenia and the USA. Int J Cancer 97: 811-818, 2002.

23. Roder JD, Stein HJ, Böttcher K, et al: Surgical therapy for gastric cancer. J Infus Chemother 5: 97-103, 1995.

24. Qiu Y, Morii E, Tomita Y, et al: Prognostic significance of pre B cell leukemia transcription factor 2 ( $\mathrm{PBX} 2$ ) expression in non-small cell lung carcinoma. Cancer Sci (In press).

25. Sobin LH and Wittekind CH: TNM classification of malignant tumours. 6th edition, Wiley-Liss, New York, 2002.

26. Qiu Y, Tomita Y, Zhang B, et al: Pre-B-cell leukemia transcription factor 1 regulates expression of valosin-containing protein, a gene involved in cancer growth. Am J Pathol 170: 152-159, 2007. 
27. Meyer-Siegler KL, Iczknowski KA, Leng L, et al: Inhibition of macrophage migration inhibitory factor or its receptor (CD74) attenuates growth and invasion of DU-145 prostate cancer cells. J Immunol 177: 8730-8739, 2006

28. Nozoe T, Saeki H, Ohga T, et al: Clinicopathological features of early esophageal squamous cell carcinoma with subsequent recurrence. Dis Esophagus 15: 145-148, 2002.

29. Dawsey SM, Wang GQ, Weinstein WM, et al: Squamous dysplasia and early esophageal cancer in the Linxian region of China: distinctive endoscopic lesions. Gastroenterology 105: 1333-1340, 1993.

30. Eloubeidi MA, Desmond R, Arguedas MR, et al: Prognostic factors for the survival of patients with esophageal carcinoma in the US: the importance of tumor length and lymph node status. Cancer 95: 1434-1443, 2002.

31. Liu C, Zhang R, Lu Y, et al: Prognostic role of lymphatic vessel invasion in early gastric cancer: A retrospective study of 188 cases. Surg Oncol (In press).

32. Vazquez-Sequeiros E, Wang L, Burgart L, et al: Occult lymph node metastases as a predictor of tumor relapse in patients with node-negative esophageal carcinoma. Gastroenterology 122: $1815-1821,2002$.

33. Longobardi E and Blasi F: Overexpression of PREP-1 in F9 teratocarcinoma cells leads to a functionally relevant increase of PBX-2 by preventing its degradation. J Biol Chem 278 . 39235-39241, 2003
34. Laurent A, Bihan R, Omilli F, et al: PBX proteins: much more than Hox cofactors. Int J Dev Biol 52: 9-20, 2008.

35. Van Dijk MA, Peltenburg LT and Murre C: Hox gene products modulate the DNA binding activity of $\mathrm{Pbx} 1$ and $\mathrm{Pbx} 2$. Mech Dev 52: 99-108, 1995.

36. Strathdee G, Holyoake TL, Sim A, et al: Inactivation of HOXA genes by hypermethylation in myeloid and lymphoid malignancy is frequent and associated with poor prognosis. Clin Cancer Res 13: 5048-5055, 2007

37. Argiropoulos B and Humphries RK: Hox genes in hematopoiesis and leukemogenesis. Oncogene 26: 6766-6776, 2007.

38. Juan M, Minhu C, Jide W, et al: Pancreatic duodenal homeobox-1 (PDX1) functions as a tumor suppressor in gastric cancer. Carcinogenesis 29: 1327-1333, 2008.

39. Potts MB, Wang DP and Cameron S: Trithorax, Hox, and TALEclass homeodomain proteins ensure cell survival through repression of the BH3-only gene egl-1. Dev Biol 329: 374-385, 2009.

40. Shears L, Plowright L, Harrington K, et al: Disrupting the interaction between HOX and PBX causes necrotic and apoptotic cell death in the renal cancer lines $\mathrm{CaKi}-2$ and 769-P. J Urol 180: 2196-2201, 2008. 